Advances in Dynamical Systems and Applications

ISSN 0973-5321, Volume 14, Number 2, pp. 189-199 (2019)

https://dx.doi.org/10.37622/ADSA/14.2.2019.189-199

\title{
Comparison of Smallest Eigenvalues for Fractional-Order Nonlocal Boundary Value Problems
}

\author{
Johnny Henderson \\ Baylor University \\ Department of Mathematics \\ Waco, TX 76798-7328 USA \\ Jeffrey T. Neugebauer \\ Eastern Kentucky University \\ Department of Mathematics and Statistics
}

\begin{abstract}
For $1<\alpha \leq 2$ a real number, we apply the theory of $u_{0}$-positive operators to establish the existence of smallest positive eigenvalues and their comparison for the $\alpha$ th-order Riemann-Liouville linear differential equations, $D_{0+}^{\alpha} y(t)+\lambda p(t) y(t)=$ 0 and $D_{0+}^{\alpha} y(t)+\sigma q(t) y(t)=0,0<t<1$, with each satisfying the nonlocal boundary conditions, $y(0)=\sum_{i=1}^{p} a_{i} y\left(\xi_{i}\right), 0<\xi_{1}<\cdots<\xi_{p}<1$, and $y(1)=$ $\sum_{j=1}^{r} b_{j} y\left(\eta_{j}\right), 0<\eta_{1}<\cdots<\eta_{r}<1$.
\end{abstract}

AMS Subject Classifications: 26A33, 34A08, 34B05, 34B09, 34B10.

Keywords: Riemann-Liouville linear differential equation, fractional order, eigenvalue comparison, nonlocal boundary condition.

\section{Introduction}

For $1<\alpha \leq 2$ a real number, we establish the existence of smallest positive eigenvalues and then their comparison for the $\alpha$ th-order Riemann-Liouville linear differential equations,

$$
D_{0+}^{\alpha} y(t)+\lambda p(t) y(t)=0, \quad 0<t<1,
$$

Received January 23, 2019; Accepted February 12, 2019

Communicated by John Graef 


$$
D_{0+}^{\alpha} y(t)+\sigma q(t) y(t)=0, \quad 0<t<1,
$$

with each satisfying the nonlocal boundary conditions,

$$
y(0)=\sum_{i=1}^{p} a_{i} y\left(\xi_{i}\right), \quad y(1)=\sum_{j=1}^{r} b_{j} y\left(\eta_{j}\right)
$$

where $p, q \in C([0,1],[0, \infty))$ and neither $p$ nor $q$ vanishes identically on any nondegenerate compact subinterval of $[0,1]$, and where $0<\xi_{1}<\cdots<\xi_{p}<1,0<\eta_{1}<\cdots<$ $\eta_{r}<1, a_{i} \geq 0,1 \leq i \leq p$, such that $0<\sum_{i=1}^{p} a_{i}<1$, and $b_{j} \geq 0,1 \leq j \leq r$, such that $0<\sum_{j=1}^{r} b_{j}<1$

Application is made of the theory of $u_{0}$-positive operators with respect to a cone in a Banach space as developed in the book by Krasnosel'skii [35] and in the book by Krein and Rutman [36]. Applications of these methods have seen extensive use in papers devoted to eigenvalue problems for differential equations, finite difference equations and dynamic equations on time scales; to name a few references, we suggest some respective groups of papers such as $[3-5,8,9,17,30,31,34,38-40,44],[2,13,14]$ and $[1,28,29,37]$.

Fractional differential equations describe many phenomena in several fields of engineering and scientific disciplines such as physics. They are now commonly viewed as better tools for the description of hereditary properties of various materials and processes than the corresponding integer-order differential equations [10, 33, 41-43]. Much current research concerning boundary value problems for fractional differential equations has involved conditions for existence of positive solutions; see, for example $[11,12,16,18,20-26,47,48]$ and references therein.

And while a few papers $[6,7,15,27,45,46]$ have been devoted to eigenvalue comparisons for fractional boundary value problems, this paper employs the Krein-Rutman theory for nonlocal (i.e., multi-point) fractional boundary value problems. The results of this paper should motivate some generalizations for nonlocal boundary value problems of higher fractional order.

Sign properties of a Green's function will be of fundamental importance in many of our arguments.

In Section 2, we state preliminary definitions and important results from the theory of $u_{0}$-positive operators with respect to a cone in a Banach space. Then, in Section 3, the results of Section 2 are applied in showing existence and comparison of smallest positive eigenvalues of (1.1)-(1.3) with smallest positive eigenvalues of (1.2)-(1.3). 


\section{Preliminaries}

In this section, we state some definitions and theorems from cone theory on which the paper's main results depend.

Let $(B,\|\cdot\|)$ be a real Banach space. A nonempty, closed subset $P$ of $B$ is a cone provided: (i) $\alpha u+\beta v \in P$, for all $\alpha, \beta \in[0, \infty)$ and for all $u, v \in P$, and (ii) $P \cap(-P)=\{0\}$. A cone $P$ is solid if $P^{\circ} \neq \emptyset$, and $P$ is reproducing, if $B=P-P$.

Remark 2.1. Krasnosel'skii [35] proved that every solid cone is reproducing.

A cone $P$ induces a partial order, $\preceq$, on the Banach space $B$ by: for $u, v \in B$, define $u \preceq v$ iff $v-u \in P$. Also, if $M, N: B \rightarrow B$ are bounded linear operators, define $M \preceq N$, if $M u \preceq N u$, for all $u \in P$.

An operator $L: B \rightarrow B$ is said to be $u_{0}$-positive with respect to $P$, if there exists $u_{0} \in P \backslash\{0\}$ such that, for each $u \in P \backslash\{0\}$, there exist $k_{1}(u), k_{2}(u) \in(0, \infty)$ such that $k_{1} u_{0} \preceq L u \preceq k_{2} u_{0}$.

The comparison of eigenvalue results for our eigenvalue problems arise from applications of the following three theorems. The proof of the first theorem can be found in many of the papers listed in the bibliography of this paper. The proof of the second theorem can be found in Krasnosel'skii's book [35], and the proof of the third theorem can be found in the paper by Keener and Travis [32].

Theorem 2.2. Let $B$ be a real Banach space and let $P \subset B$ be a solid cone. If $M$ : $B \rightarrow B$ is a linear operator such that $M: P \backslash\{0\} \rightarrow P^{\circ}$, then $M$ is $u_{0}$-positive.

Theorem 2.3. Let $B$ be a real Banach space and let $P \subset B$ be a reproducing cone. Let $M: B \rightarrow B$ be a compact, linear operator, which is $u_{0}$-positive. Then $M$ has an essentially unique eigenvector in $P$, and the corresponding eigenvalue is simple, positive and larger than the absolute value of any other eigenvalue.

Theorem 2.4. Let $B$ be a real Banach space and let $P \subset B$ be a cone. Let both $M, N: B \rightarrow B$ be bounded, linear operators, and assume that at least one of the operators is $u_{0}$-positive. If $M \preceq N, \lambda u_{1} \preceq M u_{1}$ for some $u_{1} \in P$ and some $\lambda>0$, and $N u_{2} \preceq \sigma u_{2}$ for some $u_{2} \in P$ and some $\sigma>0$, then $\lambda \leq \sigma$. Furthermore, $\lambda=\sigma$ implies $u_{1}$ is a scalar multiple of $u_{2}$.

\section{Comparison of Smallest Eigenvalues}

We remark that, from the assumptions on $a_{i}$ and $b_{i}$, we have $0<\sum_{i=1}^{p} a_{i} \xi_{i}^{\alpha-1}<1$ and $0<\sum_{j=1}^{r} b_{j} \eta_{j}^{\alpha-1}<1$. We also remark that 0 is not an eigenvalue of either (1.1)-(1.3) or (1.2)-(1.3), and so there is a Green's function, $G(t, s)$, for

$$
-D_{0+}^{\alpha} y(t)=0
$$


and satisfying the boundary conditions (1.3). We define

$$
\Delta:=\left(1-\sum_{j=1}^{r} b_{j}\right)\left(\sum_{i=1}^{p} a_{i} \xi_{i}^{\alpha-1}\right)+\left(1-\sum_{i=1}^{p} a_{i}\right)\left(1-\sum_{j=1}^{r} b_{j} \eta_{j}^{\alpha-1}\right) .
$$

From the remark above, it follows that $\Delta>0$.

Extending the arguments of Henderson and Luca [16,19], we obtain by direct computation that the Green's function for (3.1)-(1.3) is given by,

$$
\begin{aligned}
G(t, s):= & g(t, s)+\frac{1}{\Delta}\left[\left(1-t^{\alpha-1}\right)\left(1-\sum_{j=1}^{r} b_{j}\right)+\sum_{j=1}^{r} b_{j}\left(1-\eta_{j}^{\alpha-1}\right)\right] \sum_{i=1}^{p} a_{i} g\left(\xi_{i}, s\right) \\
& +\frac{1}{\Delta}\left[t^{\alpha-1}\left(1-\sum_{i=1}^{p} a_{i}\right)+\sum_{i=1}^{p} a_{i} \xi_{i}^{\alpha-1}\right] \sum_{j=1}^{r} b_{j} g\left(\eta_{j}, s\right),
\end{aligned}
$$

for $(t, s) \in[0,1] \times[0,1]$, and where

$$
g(t, s)=\frac{1}{\Gamma(\alpha)} \begin{cases}t^{\alpha-1}(1-s)^{\alpha-1}-(t-s)^{\alpha-1}, & 0 \leq s \leq t \leq 1 \\ t^{\alpha-1}(1-s)^{\alpha-1}, & 0 \leq t \leq s \leq 1\end{cases}
$$

Properties of $G(t, s)$ that we will subsequently use include:

- For each fixed $0<s<1$, as a function of $t, G(t, s)$ satisfies the boundary conditions (1.3).

- $G(t, s) \geq g(t, s) \geq 0$ on $[0,1] \times[0,1]$.

- $G(t, s)>g(t, s)>0$ on $(0,1) \times(0,1)$.

In applying Theorems $2.2-2.4$, we will define the Banach space

$$
B:=C[0,1]
$$

with norm

$$
\|u\|:=\max _{0 \leq t \leq 1}|u(t)|
$$

and we define the cone

$$
P:=\{u \in B \mid u(t) \geq 0 \text { on }[0,1]\} .
$$

Lemma 3.1. The cone P has nonempty interior and $Q:=\{v \in B \mid v(t)>0$ on $[0,1]\} \subseteq$ $P^{\circ}$.

The following is immediate by Remark 2.1.

Corollary 3.2. The cone $P$ is reproducing. 
Now, we define linear operators $M, N: B \rightarrow B$ by

$$
M u(t):=\int_{0}^{1} G(t, s) p(s) u(s) d s, t \in[0,1]
$$

and

$$
N u(t):=\int_{0}^{1} G(t, s) q(s) u(s) d s, t \in[0,1],
$$

where $G(t, s)$ is the Green's function for (3.1)-(1.3).

Remark 3.3. By making the standard arguments, it is straightforward that both $M$ and $N$ are compact operators.

Remark 3.4. At this point we observe that

$$
\Lambda u(t)=M u(t)=\int_{0}^{1} G(t, s) p(s) u(s) d s, t \in[0,1]
$$

if and only if

$$
u(t)=\frac{1}{\Lambda} \int_{0}^{1} G(t, s) p(s) u(s) d s, t \in[0,1],
$$

if and only if

$$
-D_{0+}^{\alpha} u(t)=\frac{1}{\Lambda} p(t) u(t), 0<t<1, \text { and } u(0)=\sum_{i=1}^{p} a_{i} u\left(\xi_{i}\right), u(1)=\sum_{j=1}^{r} b_{j} u\left(\eta_{j}\right) .
$$

Namely, the eigenvalues of (1.1)-(1.3) are multiplicative reciprocals of the eigenvalues of $M$, and conversely. Similarly, the eigenvalues of (1.2)-(1.3) are multiplicative reciprocals of the eigenvalues of $N$, and conversely.

Theorem 3.5. The bounded linear operators $M$ and $N$ are $u_{0}$-positive.

Proof. In proving the result for the operator $M$, it suffices, by Theorem 2.2, to show that $M: P \backslash\{0\} \rightarrow P^{\circ}$.

We first show that $M: P \rightarrow P$. Choose $u \in P$. Each of $u(t), G(t, s)$ and $p(t)$ is nonnegative valued, and so it follows that, for $0 \leq t \leq 1$,

$$
M u(t)=\int_{0}^{1} G(t, s) p(s) u(s) d s \geq 0 .
$$

Therefore, $M u \in P$.

Now, choose $u \in P \backslash\{0\}$. Then by the assumptions on $p(t)$ stated in the Introduction, there exists $[c, d] \subseteq[0,1]$ such that $u(t) p(t)>0$ on $[c, d]$. Moreover, from the properties above for the Green's function, $G(t, s)>0$ on $(0,1) \times(0,1)$. Define

$$
z(t):=\int_{0}^{1} G(t, s) p(s) u(s) d s
$$


Then $z \in P$, and for $0<t<1$,

$$
z(t) \geq \int_{c}^{d} G(t, s) p(s) u(s) d s>0 .
$$

In addition, from properties of the Green's function, $z(t)$ satisfies the boundary conditions (3). Coupled with the stated properties in the Introduction on $a_{i}, i=1, \ldots, p$, and on $b_{j}, j=1, \ldots, r$,

$$
z(0)=\sum_{i=1}^{p} a_{i} z\left(\xi_{i}\right)>0 \text { and } z(1)=\sum_{j=1}^{r} b_{j} z\left(\eta_{j}\right)>0 .
$$

In particular, $z(t)>0$ on $[0,1]$. That is, $z \in Q \subset P^{\circ}$, and so $M: P \backslash\{0\} \rightarrow P^{\circ}$. In a similar way, $N$ is also $u_{0}$-positive.

Theorem 3.6. . The operator $M$ (and $N$ ) has an eigenvalue that is simple, positive, and larger than the absolute value of any other eigenvalue, with an essentially unique eigenvector that can be chosen to belong to $P^{\circ}$.

Proof. $M$ is a $u_{0}$-positive, compact linear operator, and so by Theorem 2.3, $M$ has an essentially unique eigenvector, say $u \in P$, and an eigenvalue $\Lambda$ having the properties in the statement of this theorem. Since $u \neq 0, M u \in Q \subset P^{\circ}$, and so $u=M\left(\frac{1}{\Lambda} u\right) \in$ $P^{\circ}$.

Theorem 3.7. Let $p(t) \leq q(t)$ on $[0,1]$. Let $\Lambda$ and $\Sigma$ be the eigenvalues of Theorem 3.6 corresponding to $M$ and $N$, respectively, with associated essentially unique eigenvectors, $u_{1}$ and $u_{2}$, that belong to $P^{\circ}$. Then, $\Lambda \leq \Sigma$, and $\Lambda=\Sigma$ if and only if $p(t)=q(t)$, for all $t \in[0,1]$.

Proof. With $p(t) \leq q(t)$ on $[0,1]$, then, for any $u \in P$ and for any $t \in[0,1]$,

$$
(N u-M u)(t)=\int_{0}^{1} G(t, s)[q(s)-p(s)] u(s) d s \geq 0,
$$

and so $M \preceq N$. By Theorem 2.4, then $\Lambda \leq \Sigma$.

For the last part of the this theorem, if $p(t) \equiv q(t)$, then of course $\Lambda=\Sigma$. Next, for the purpose of contrapositive argument, suppose $p(t) \not \equiv q(t)$. Then there is some subinterval $[a, b] \subset[0,1]$ on which $p(t)<q(t)$. It follows that $(N-M)\left(u_{1}\right) \in Q \subset P^{\circ}$. So there exists an $\epsilon>0$ such that $(N-M)\left(u_{1}\right)-\epsilon u_{1} \in P^{\circ}$, from which

$$
\Lambda u_{1}+\epsilon u_{1}=M u_{1}+\epsilon u_{1} \preceq N u_{1} .
$$

This implies $(\Lambda+\epsilon) u_{1} \preceq N u_{1}$, and since $N \preceq N$ and $N u_{2}=\Sigma u_{2}$, Theorem 2.4 implies that $\Lambda+\epsilon \leq \Sigma$, or $\Lambda<\Sigma$. 
By Remark 3.4 and Theorems 3.6 and 3.7, we can now state our main result concerning existence and comparison of smallest positive eigenvalues for (1.1)-(1.3) and (1.2)-(1.3).

Theorem 3.8. Assume the hypotheses of Theorem 3.7. Then there exist smallest positive eigenvalues $\lambda$ and $\sigma$ of (1.1)-(1.3) and (1.2)-(1.3), respectively, each of which is simple, positive, and less than the absolute value of any other eigenvalue of the corresponding problem, and the eigenvectors corresponding to $\lambda$ and $\sigma$ may be chosen to belong to $P^{\circ}$. Finally, $\lambda \geq \sigma$, and $\lambda=\sigma$ if and only if $p(t)=q(t)$, for all $t \in[0,1]$.

\section{References}

[1] C. J. Chyan, J. M. Davis, J. Henderson and W. K. C. Yin, Eigenvalue comparisons for differential equations on a measure chain, Electron. J. Differential Equations 1998, No. 35, 7 pp.

[2] J. M. Davis, P. W. Eloe and J. Henderson, Comparison of eigenvalues for discrete Lidstone boundary value problems, Dynam. Systems Appl. 8 (1999), 381-388.

[3] P. W. Eloe and J. Henderson, Comparison of eigenvalues for a class of two-point boundary value problems, Appl. Anal. 34 (1989), 25-34.

[4] P. W. Eloe and J. Henderson, Focal points and comparison theorems for a class of two-point boundary value problems, J. Differential Equations 103 (1993), 375386.

[5] P. W. Eloe and J. Henderson, Focal point characterizations and comparisons for right focal differential operators, J. Math. Anal. Appl. 181 (1994), 22-34.

[6] P. W. Eloe and J. T. Neugebauer, Existence and comparison of smallest eigenvalues for a fractional boundary value problem, Electron. J. Differential Equations 2014, No. 43, 10 pp.

[7] P. W. Eloe and J. T. Neugebauer, Smallest eigenvalues for a right focal boundary value problem, Fract. Calc. Appl. Anal. 19 (2016), no. 1, 11-18.

[8] K. M. Fick, Comparison of eigenvalues for $2 n$th order eigenvalue problems, Commun. Appl. Anal. 8 (2004), no. 1, 17-26.

[9] R. D. Gentry and C. C. Travis, Comparison of eigenvalues associated with linear differential equations of arbitrary order, Trans. Amer. Math. Soc. 223 (1976), 167179.

[10] C. Goodrich and A. C. Peterson, Discrete Fractional Calculus, Springer, Cham, 2015. 
[11] J. R. Graef, L. Kong, Q. Kong and M. Wang, Uniqueness of positive solutions of fractional boundary value problems with non-homogeneous integral boundary conditions, Fract. Calc. Appl. Anal. 15 (2012), No. 3, 509-528.

[12] S. Hamani, J. Henderson and J. J. Nieto, Boundary value problem for fractional differential equations with nonlocal conditions in Banach spaces, Fixed Point Theory 18 (2017), no. 2, 591-600.

[13] D. Hankerson and J. Henderson, Comparison of eigenvalues for $n$-point boundary value problems for difference equations, Differential Equations (Colorado Springs, CO, 1989) 127, Lecture Notes in Pure and Applied Mathematics, Dekker, New York, NY, 1991, 203-208.

[14] D. Hankerson and A. C. Peterson, Comparison of eigenvalues for focal point problems for $n$th order difference equations, Differential Integral Equations 3 (1990), no. $2,363-380$.

[15] J. Henderson and N. Kosmatov, Eigenvalue comparison for fractional boundary value problems with the Caputo derivative, Fract. Calc. Appl. Anal. 17 (2014), no. $3,872-880$.

[16] J.Henderson and R. Luca, Positive solutions for a system of nonlocal fractional boundary value problems, Fract. Calc. Appl. Anal. 16 (2013), No. 4, 985-1008.

[17] J. Henderson and R. Luca, Comparison of smallest eigenvalues for certain third order boundary value problems, PanAmer. Math. J. 24 (2014), no. 3, 75-81.

[18] J. Henderson and R. Luca, Positive solutions for systems of nonlinear second-order multipoint boundary value problems, Math Methods Appl. Sci. 37 (2014), no. 16, 2502-2516.

[19] J. Henderson and R. Luca, On a system of Riemann-Liouville boundary value problems, Comm. Appl. Nonlinear Anal. 23 (2016), No. 2, 1-19

[20] J. Henderson and R. Luca, Boundary Value Problems for Systems of differential, Difference and Fractional equations. Positive Solutions, Elsevier, Amsterdam, 2016.

[21] J. Henderson and R. Luca, Existence of positive solutions for a system of semipositone fractional boundary value problems, Electron. J. Qual. Theory Differ. Equ. 2016, Paper No. 22, 28 pp.

[22] J. Henderson and R. Luca, Systems of Riemann-Liouville fractional equations with multi-point boundary conditions, Appl. Math. Comput. 309 (2017), 303-323. 
[23] J. Henderson and R. Luca, Existence of positive solutions for a singular fractional boundary value problem, Nonlinear Anal. Model. Control 22 (2017), no. 1, 99114.

[24] J. Henderson and R. Luca, Positive solutions for a system of coupled fractional boundary value problems, Lith. Math. J. 58 (2018), no. 1, 15-32.

[25] J. Henderson, R. Luca and A. Tudorache, Existence of positive solutions for a system of fractional boundary value problems. Differential and Difference Equations with Applications, pp. 349-357, Springer Proc. Math. Stat., 164, Springer, Cham, 2016.

[26] J. Henderson, R. Luca and A. Tudorache, Positive solutions for a system of fractional differential equations with multi-point boundary conditions, ROMAI J. 13 (2017), no. 2, 85-100.

[27] J. Henderson and J. T. Neugebauer, Smallest eigenvalues for a fractional difference equation with right focal boundary conditions, J. Difference Equ. Appl. 23 (2017), no. $8,1317-1323$.

[28] J. Henderson and K. R. Prasad, Comparison of eigenvalues for Lidstone boundary value problems on a measure chain, Comput. Math. Appl. 38 (1999), no. 11-12, $55-62$.

[29] J. Hoffacker, Green's functions and eigenvalue comparisons for a focal problem on time scales, Comput. Math. Appl. 45 (2003), 1339-1368.

[30] B. Karna, Eigenvalue comparisons for three-point boundary value problems, Comm. Appl. Nonlinear Anal. 12 (2005), no. 3, 83-91.

[31] B. Karna, E. R. Kaufmann and J. Nobles, Comparison of eigenvalues for a fourthorder four-point boundary value problem, Electron. J. Qual. Theory Differ. Equ. 2005, No. 15, 9 pp.

[32] M. Keener and C. C. Travis, Positive cones and focal points for a class of $n$th order differential equations, Trans. Amer. Math. Soc. 237 (1978), 331-351.

[33] A. A. Kilbas, H. M. Srivastava and J. J. Trujillo, Theory and Applications of Fractional Differential Equations, North-Holland Mathematics Series, 204, Elsevier Science B. V., Amsterdam, 2006.

[34] S. S. King and J. T. Neugebauer, Smallest eigenvalues, extremal points, and positive solutions of a fourth order three point boundary value problem, Dynam. Systems Appl. 23 (2014), no. 4, 699-713. 
[35] M. A. Krasnosel'skii, Positive Solutions of Operator Equations, P. Noordhoff Ltd., Groningen, The Netherlands, 1964.

[36] M. G. Krein and M. A. Rutman, Linear Operators Leaving a Cone Invariant in a Banach Space, in American Mathematical Society Translations, Series 1, Providence, 1962.

[37] B. A. Lawrence and D. T. Reid, Comparison of eigenvalues for Sturm-Liouville boundary value problems on a measure chain, Comput. Math. Appl. 45 (2003), no. 6-9, 1319-1326.

[38] C. Nelms Jr, Comparison of smallest eigenvalues for certain fifth order boundary value problems, Adv. Dyn. Syst. Appl. 10 (2015), no. 1, 77-84.

[39] J. T. Neugebauer, Methods of extending lower order problems to higher order problems in the context of smallest eigenvalue comparisons, Electron. J. Qual. Theory Differ. Equ. 2011, No. 99, 16 pp.

[40] J. T. Neugebauer, Existence and comparison of smallest eigenvalues and extremal points for a three point boundary value problem, Math. Sci. Res. J. 16 (2012), no. 9, 222-233.

[41] I. Podlubny, Fractional Differential Equations. An Introduction to Fractional Derivatives, Fractional Differential Equations, to Methods of Their Solution and Some of Their Applications, Mathematics in Science and Engineering, 198, Academic Press, San Diego, 1999.

[42] J. Sabatier, O. P. Agrawal and J. A. T. Machado (Eds.) Advances in Fractional Calculus: Theoretical Developments and Applications in Physics and Engineering, Springer, Dordrecht, 2007.

[43] S. G. Samko, A. A. Kilbas and O. I. Marichev, Fractional Integrals and Derivatives. Theory and Applications, Gordon and Breach, Yverdon, 1993.

[44] C. C. Travis, Comparison of eigenvalues for linear differential equations of order 2n, Trans. Amer. Math. Soc. 177 (1973), 363-374.

[45] A. Yang and J. Henderson, Comparison of smallest eigenvalues for fractional difference equations, Enlightenment Pure Appl. Math. 2 (2016), 161-170.

[46] A. Yang, L. Zhang and J. Henderson, Comparison of smallest eigenvalues for right focal Atici-Eloe fractional difference equations, J. Korean Soc. Math. Educ. Ser. B Pure Appl. Math. 24 (2017), no. 4, 191-200.

[47] C. Yuan, Two positive solutions for (n?1,1)-type semipositone integral boundary value problems for coupled systems of nonlinear fractional differential equations, Commun. Nonlinear Sci. Numer. Simulat. 17 (2012), No. 2, 930-942. 
[48] C. Yuan, D. Jiang, D. O’Regan, R. P. Agarwal, Multiple positive solutions to systems of nonlinear semipositone fractional differential equations with coupled boundary conditions, Electron. J. Qual. Theory Differ. Equ. 2012, No. 13, 1-17. 
